\section{The cost-effectiveness of routine testing for Lynch syndrome in newly diagnosed patients with colorectal cancer in the United States: corrected estimates}

To the Editor: We compliment Severin et al. ${ }^{1}$ at the Helmholtz Center in Germany on their sophisticated cost-effectiveness analysis (CEA) of testing strategies for Lynch syndrome (LS) in Germany. The authors make a persuasive case that offering testing to newly diagnosed patients with colorectal cancer (CRC) is less likely to be cost-effective in Germany than in the United States. This is due, in part, to differences in reimbursements for genetic tests, the smaller number of first-degree relatives available to be tested, and the lower level of willingness to undergo mutation testing.

The incremental cost-effectiveness ratios (ICERs) in the Helmholtz model are much higher than previously published estimates from the United States. For example, in 2010, we estimated a net cost of less than $\$ 25,000$ per life-year gained (LYG) for universal offers of testing to newly diagnosed patients with CRC using a model developed at the Centers for Disease Control and Prevention (CDC). ${ }^{2}$ That ICER compares with more than $\$ 300,000$ per LYG for the same strategy reported by Severin et al. ${ }^{1}$ The major reason for that difference is that in the Helmholtz model the most favorable strategy restricts testing to patients who meet the Revised Bethesda Guidelines, which is assumed to detect approximately $88 \%$ of cases of LS. By contrast, our model did not consider a family history-based testing strategy because of lack of evidence that it would be practical in a routine clinical setting to collect and interpret the detailed information about family history of cancer necessary to apply those guidelines. We did compare a strategy using the first criterion, offering testing to probands younger than 50 years of age, and found that universal testing was cost-effective even compared with offering testing only to younger probands. ${ }^{2}$

In addition, Severin et al. ${ }^{1}$ found different LYG per LS carrier undergoing intensive surveillance ( 0.52 in their Table 1 versus 1.07 in our Table 1; 2,197 relatives gain 2,347 LYG in strategy 1 ). Severin et al. ${ }^{1}$ shared helpful comments with us regarding our model, which they reviewed in detail. We take this opportunity to acknowledge some errors in our model and to report corrected estimates.

First, our CDC model did not multiply incidence rates by general US life table survival probabilities to account for attrition from other causes of death. Correcting that error reduces the gain in LYG by $5 \%$, to 1.02 per adherent carrier. Second, although we stated that the LS carriers who develop CRC were assumed to have better survival rates compared with other patients with CRC regardless of use of colonoscopic surveillance, the case-fatality rate for the general population with CRC was incorrectly applied to LS carriers who do not follow colonoscopic surveillance. After correcting that error, the LYG per adherent carrier is reduced to 0.91 .

In modifying our model, we also changed two assumptions to be more conservative in the sense that the revised model projects fewer lives saved from LS testing. First, we now follow Severin et al. ${ }^{1}$ in using relative age-specific incidence rates of CRC in LS mutation carriers from a study published in $2011,{ }^{3}$ in which it was found that $31.0 \%$ of cumulative CRC incidence in LS carriers occurs prior to age 50 , compared with $46.5 \%$ in our original model. In addition, we replaced our original assumption of $62 \%$ reduction in CRC incidence with frequent surveillance with the $59 \%$ reduction reported in our source for that parameter. ${ }^{4}$ When we make both of those substitutions in our model, the estimate of LYG per adherent carrier is further reduced to 0.80 . Those changes together account for approximately one-half of the overall gap in projected survival between the Helmholtz and CDC models. Finally, we corrected an error in the formula for the calculation of the costs associated with complications of colonoscopy. The cumulative effect of these various model revisions is to raise the base-case ICER estimate of strategy 1 (universal testing relative to no testing) from $\$ 22,552$ per LYG in our published results to $\$ 31,391$ per LYG, an increase of $39 \%$. Although higher in absolute terms, our revised ICER is still low relative to usual cost-effectiveness thresholds of $\$ 50,000$ $\$ 100,000$ per LYG. In addition, LS carriers younger than age 50 account for a smaller portion of LS-related CRC than we originally assumed. Consequently, universal offer of testing for LS in newly diagnosed patients with CRC in the United States still appears cost-effective.

We thank Severin et al. ${ }^{1}$ for their close attention to detail, which helped us to correct and update our previously published estimates of the cost-effectiveness of offering testing for LS to probands with newly diagnosed CRC in the United States. Critical analysis (and re-analysis) of epidemiologic assumptions underlying CEA models is crucial to allow for an accurate and objective assessment of cost-effectiveness estimates. It is unfortunate that in-depth, independent analyses of CEA models and their underlying assumptions are rarely conducted.

\section{ACKNOWLEDGMENTS}

The findings and conclusions in this report are those of the authors and do not necessarily represent the official position of the Centers for Disease Control and Prevention.

\section{DISCLOSURE}

The authors declare no conflict of interest.

Scott D. Grosse, PhD ${ }^{1}$, Glenn E. Palomaki, BS ${ }^{2}$, Mercy Mvundura, $P h D^{3}$ and Heather Hampel, $M S^{4}$

\footnotetext{
${ }^{1}$ National Center on Birth Defects and Developmental Disabilities, Centers for Disease Control and Prevention, Atlanta, Georgia, USA; ${ }^{2}$ Department of Pathology and Laboratory Medicine, Women and Infants Hospital/Alpert Medical School at Brown University, Providence, Rhode Island, USA; ${ }^{3}$ Program on Appropriate Technology in Health, Seattle, Washington, USA; ${ }^{4}$ Division of Human Genetics, Department of Internal Medicine, The Ohio State University Comprehensive Cancer Center, Columbus, Ohio, USA. Correspondence: Scott Grosse (sgrosse@cdc.gov)
} 


\section{REFERENCES}

1. Severin F, Stollenwerk B, Holinski-Feder E, et al. Economic evaluation of genetic screening for Lynch syndrome in Germany. Genet Med; e-pub ahead of print 8 January2015.

2. Mvundura M, Grosse SD, Hampel H, Palomaki GE. The cost-effectiveness of genetic testing strategies for Lynch syndrome among newly diagnosed patients with colorectal cancer. Genet Med 2010;12: 93-104.
3. Bonadona V, Bonaïti B, Olschwang S, et al.; French Cancer Genetics Network. Cancer risks associated with germline mutations in $\mathrm{MLH1}, \mathrm{MSH} 2$, and MSH6 genes in Lynch syndrome. JAMA 2011;305:2304-2310.

4. Stupart DA, Goldberg PA, Algar U, Ramesar R. Cancer risk in a cohort of subjects carrying a single mismatch repair gene mutation. Fam Cancer 2009;8:519-523.

doi:10.1038/gim.2015.53 